\title{
Self-organized growth of bamboo-like carbon nanotube arrays for field emission properties
}

\author{
Balaji Padya $\cdot$ Dipankar Kalita $\cdot$ P. K. Jain $\cdot$ \\ G. Padmanabham $\cdot$ M. Ravi $\cdot$ K. S. Bhat
}

Received: 4 November 2011/Accepted: 21 March 2012/Published online: 3 April 2012

(C) The Author(s) 2012. This article is published with open access at Springerlink.com

\begin{abstract}
Well-aligned nitrogen-doped carbon nanotube (N-CNTs) film was fabricated on silicon substrate by thermal chemical vapor deposition process with varying the growth temperature. The effect of growth temperature on morphology, microstructure and crystallinity for the growth of N-CNTs was studied. At all growth temperatures, the bamboo-like morphology of graphene layers with compartments in CNTs were observed in transmission electron microscope micrographs. The doping level and the type of nitrogen-related moieties were determined by X-ray photoelectron spectroscopy analysis. The compartment distance decreases with increase in nitrogen doping level in hexagonal graphite network. The increase in nitrogen doping level in N-CNTs will lead to decrease in crystallinity and in-plane crystallite size. Field emission study of nitrogen-doped carbon nanotubes grown at optimum parameters showed that they are good emitters with a turn-on and threshold field of 0.3 and $1.6 \mathrm{~V} / \mu \mathrm{m}$, respectively. The maximum current density was observed to be $18.8 \mathrm{~mA} / \mathrm{cm}^{2}$ at the electric field of $2.1 \mathrm{~V} / \mu \mathrm{m}$. It is considered that the enhanced field emission performance of doped nanotube is due to the presence of lone pairs of electrons on nitrogen atom that supplies more electrons to the conduction band.
\end{abstract}

Keywords Bamboo-like carbon nanotubes . Field emission - Turn-on field · Threshold field

B. Padya · D. Kalita · P. K. Jain $(\bowtie) \cdot$ G. Padmanabham Centre for Carbon Materials, International Advanced Research Centre for Powder Metallurgy and New Materials (ARCI), Hyderabad 500 005, India

e-mail: pkjain@arci.res.in

M. Ravi · K. S. Bhat

Microwave Tubes Research and Development Centre (MTRDC), Banglore 560 013, India

\section{Introduction}

Miniaturization of nano-scale devices forced researchers to discover advanced materials with unique bulk and surface properties. Carbon nanotube (CNT) is one of the promising materials having exceptional properties for potential use in scanning probe sensors (Hafner et al. 1999), cold cathode flat panel display (Choi et al. 1999), fuel cells (Reddy et al. 2009), supercapacitors (Niu et al. 1997) and vacuum nanoelectronics. Compared to pristine CNTs, the synthesis of nitrogen-doped carbon nanotubes (N-CNTs) have gained growing interest to tailor the electronic (Terrones et al. 2002; Ghosh et al. 2010; Lim et al. 2006), electrical (Ibrahim et al. 2010) and mechanical properties (Ganesan et al. 2010) with the controlled process parameters aid. The electrical and electronic properties of the CNTs can be modified to a large extent by doping the CNTs. It has been predicted that the $\mathrm{N}$-CNTs have n-type conductivity, low resistance and excellent electron field emission property (Czerw et al. 2001). Incorporation of $\mathrm{N}$ in carbon network will lead to the shift of the localized state to the Fermi level, and the significant contribution of the coupled state near the Fermi level (Czerw et al. 2001). In wide range of applications of CNTs, field emission is of paramount interest in which electrons are emitted from a solid material by quantum mechanical tunneling when electric field is applied in high vacuum. CNTs are considered to be one of the best candidates for the field emission due to their atomically sharp tips with very high aspect ratio. The field emission property of the material is dependent on electronic properties. The electronic property of the N-CNTs depends on the dopant concentration and it is a relatively facile method to tune this property by changing the dopant concentration into the bulk material.

This paper mainly focused on feasible process to develop the organized growth of highly aligned N-CNT 
arrays with nitrogen content modulated to tailor the field emission property.

\section{Experimental procedure}

Nitrogen-doped CNTs were synthesized by injection chemical vapor deposition (CVD) on silicon substrate. The silicon substrate was cleaned with acetone and placed at the center of the furnace. The chamber was evacuated and then filled with argon gas to create the inert atmosphere inside the chamber before injecting the precursors. The precursors used were acetonitrile (Trident Fine Chemicals and laboratories, $99.8 \%$ ) and Ferrocene (Merck, $98 \%$ ). Acetonitrile was the dual source for carbon and nitrogen while ferrocene, on pyrolysis, provided iron which acts as the catalyst for CNT growth. The catalyst and precursor solution was made with the catalyst concentration of $10 \mathrm{mg} / \mathrm{ml}$ and the solution was injected at the rate of $0.5 \mathrm{ml} / \mathrm{min}$. The precursor was vaporized at $170{ }^{\circ} \mathrm{C}$ and carried towards the silicon substrate by the carrier gas. The synthesis was carried out at the three different growth temperatures 800, 850 and $900{ }^{\circ} \mathrm{C}$ with argon gas flow rate of $180 \mathrm{sccm}$.

The alignment and microstructure of as-grown CNTs were characterized using scanning electron microscope (SEM, Model S-4300S E/N, Hitachi, Japan) and transmission electron microscope (TEM, Model FEI Technai $\mathrm{G}^{2}$ S-Twin). Raman spectroscopy (Lab Ram HR 800 Raman spectrometer) was carried out using $514.5 \mathrm{~nm}$ green line of Ar+ ion laser to study the crystallinity and the disorder in CNTs. The measurement of elemental composition was carried out using X-ray photoelectron spectroscopy (XPS, Omicron with $\mathrm{Al} \mathrm{K} \mathrm{K}_{\alpha}$ 1,486.8 eV radiation source). N-CNTs arrays thin film deposited on silicon wafer was used as the cold cathode and stainless steel plate as counter electrode was placed at a distance of $1 \mathrm{~mm}$ for field emitters. The field emission properties of the deposited N-CNTs were characterized in high vacuum environment with a base pressure of about $6.3 \times 10^{-7}$ mbar. Programmable Keithley 248 high voltage power supply with low output ripple for precision sourcing and flexible operation was used for sourcing the voltage (up to 3,000 V) and measuring the current (with pA sensitivity), allowing the characterization of the current-voltage $(I-V)$ behavior.

\section{Results and discussion}

Microstructure analysis

Figure 1 shows the SEM and TEM images of N-CNT arrays grown at different growth temperatures. It can be observed that the average diameter of the N-CNTs grown at 800,850 and $900{ }^{\circ} \mathrm{C}$ are 28,65 and $33 \mathrm{~nm}$, respectively. $\mathrm{N}-\mathrm{CNT}$ arrays grown at the growth temperature $850{ }^{\circ} \mathrm{C}$ are well aligned having the length up to $50 \mu \mathrm{m}$. At 800 and $900{ }^{\circ} \mathrm{C}$ the CNTs are short and not highly aligned. It indicates that the carbon source that decomposed at $850{ }^{\circ} \mathrm{C}$ was able to provide the more amount of precursor for the nucleation of the carbon nanotubes leading to the increase in length as compared to other growth temperatures. Similar phenomenon was observed by other groups (He et al. 2005). The change in their microstructure by nitrogen incorporation was investigated by TEM studies. Nitrogendoped CNTs exhibit very distinct morphologies, significantly different from undoped counterparts. The undoped CNTs are continuously hollow throughout their length. Doped CNTs exhibited bamboo-shaped morphology and each carbon nanotube is separated into a series of compartments with corrugated walls (Jang et al. 2004). This is caused by nitrogen atoms embedded in graphitic lattice, including the curvature of nanotubes due to the different bond length of $\mathrm{C}-\mathrm{N}$ and $\mathrm{C}-\mathrm{C}$. The difference in the bond length will induce the strain in the hexagonal graphite network and the graphene sheets will try to bend due to pulsating effect. The introduction of such defects disrupts the planar hexagonal arrangement of carbon atoms in CNTs and results in the rough surface of doped CNTs. The increased nitrogen concentration induces more defective graphene sheets. As a result the graphene sheets are easily buckled, facilitating cross-linking between the graphene planes through $\mathrm{sp}^{3}$ coordinated carbons. Through the N-sp ${ }^{2}$ $\mathrm{C}$ bond, nitrogen is incorporated in the graphene sheets without changing the graphitic structure. TEM micrographs depicted that the length of the compartment in N-CNTs keeps on decreasing as the nitrogen content in hexagonal network increases. As the nitrogen content increases, the buckling of the graphene sheets will take place and try to stabilize themselves by framing the curved layers, i.e., internal capping. The distance between internal capping of CNTs grown at 800,850 and $900{ }^{\circ} \mathrm{C}$ was found to be 43 , 60 and $20 \mathrm{~nm}$, respectively.

\section{X-ray photoelectron spectroscopy}

The composition and the functional moieties present in $\mathrm{N}-\mathrm{CNTs}$ were analyzed by using the XPS. It provides detailed information on the occupied electronic levels and chemical binding states of individual atoms. The Gaussian multi-peak reveals the individual nitrogen moieties bonded to the carbon atoms in CNTs. XPS-wide spectrum showed strong peak at 285, 401 and $530 \mathrm{eV}$ which can be assigned to $\mathrm{C} 1 \mathrm{~s}$ from $\mathrm{sp}^{2}$ hybridized carbon in the nanotubes; the carbon atoms on the edge plane can be easily oxidized to oxygen-containing functionalities and nitrogen incorporated in carbon network, respectively. The asymmetric N1s 

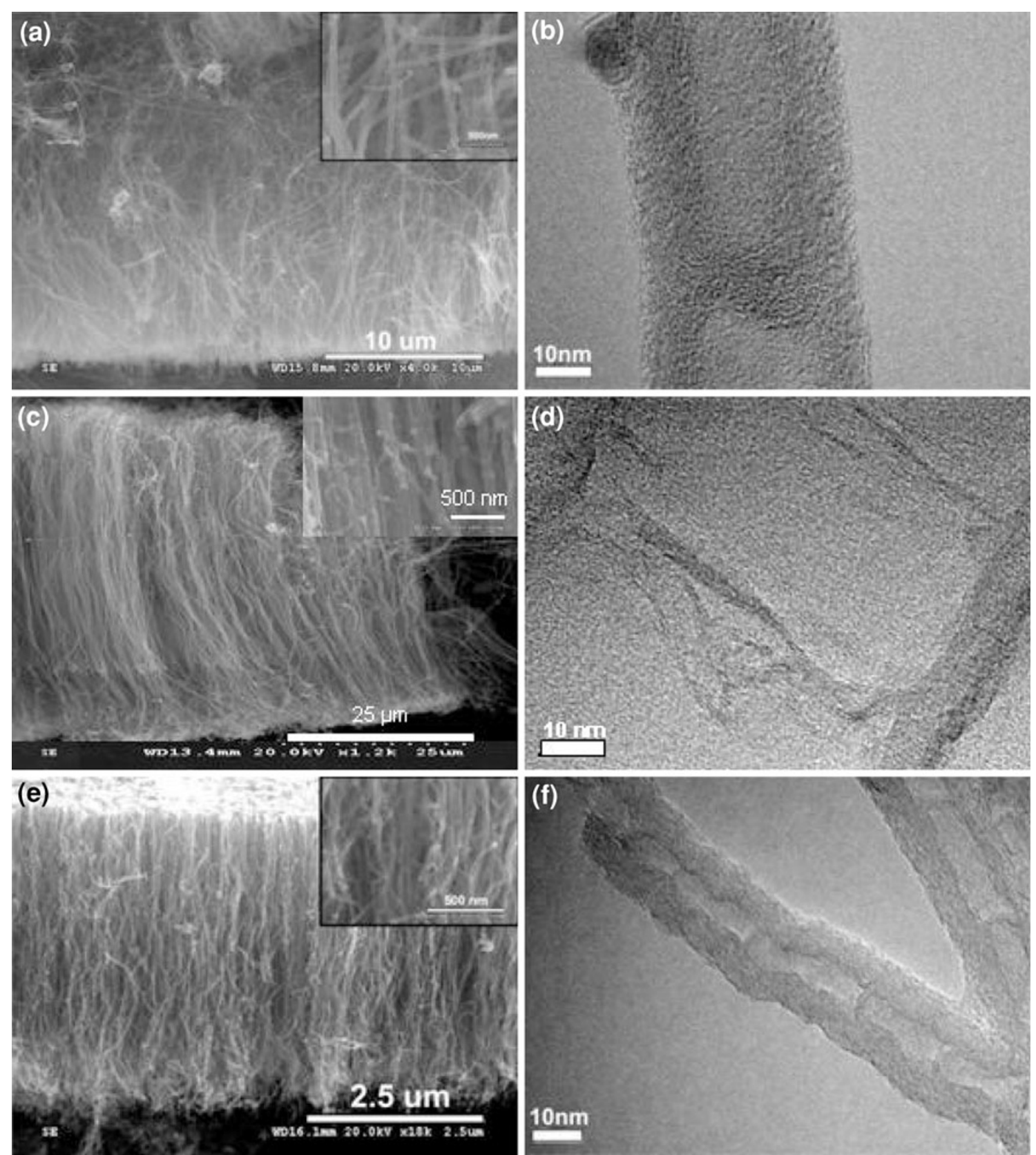

Fig. 1 Typical SEM and TEM micrographs of NCNT arrays grown at different growth temperatures at $800{ }^{\circ} \mathrm{C}(\mathbf{a}, \mathbf{b})$, at $850{ }^{\circ} \mathrm{C}(\mathbf{c}, \mathbf{d})$ and at $900{ }^{\circ} \mathrm{C}(\mathbf{e}, \mathbf{f})$

peak in narrow spectrum centered at $401 \mathrm{eV}$ can be deconvoluted into four peaks at 398.5, 401, 403 and $405 \mathrm{eV}$ (Liu et al. 2010). They are ascribed as pyridine-like nitrogen $(\mathrm{pN})$, graphitic-like nitrogen $(\mathrm{gN})$, oxidized nitrogen and molecular nitrogen. $398.5 \mathrm{eV}$ peak is assigned to a tetragonal nitrogen phase bonded to a $\mathrm{sp}^{3}$ hybridized carbon, $401 \mathrm{eV}$ assigned to a trigonal nitrogen phase bonded to a $\mathrm{sp}^{2}$ coordinated carbon, $403 \mathrm{eV}$ is assigned to oxidized $\mathrm{N}$ species or oxygen molecules physisorbed onto the rough surface and $405 \mathrm{eV}$ is assigned to molecular nitrogen molecules adsorbed or intercalated at the carbon walls or trapped in the compartments.

With decreasing the growth temperature, the XPS peak intensities for the $\mathrm{N}-\mathrm{sp}^{2} \mathrm{C}$ bond decreased, whereas those for the $\mathrm{N}-\mathrm{sp}^{3} \mathrm{C}$ bond increased, accounting for the deteriorated crystallinity of the graphene sheets. The $\mathrm{N}-\mathrm{sp}^{3} \mathrm{C}$ bonds were found to be more abundant than the $\mathrm{N}-\mathrm{sp}^{2} \mathrm{C}$ bonds around $4 \%$ nitrogen concentration. The percentage of nitrogen in CNTs was calculated by taking into account the individual areas of C1s and N1s. The formula used is 
$N \%=[N \times 100 /(C+N)]$. The nitrogen content was found to decrease from 4.30 to $3.44 \%$ when the growth temperature was increased from 800 to $850{ }^{\circ} \mathrm{C}$, whereas, the $N \%$ was increased to $12.34 \%$ at $900{ }^{\circ} \mathrm{C}$ growth temperature. The abnormal behavior in increase in nitrogen content was observed as the growth temperature increased and the reason for this is yet to be understood. The graphite type nitrogen $\left(\mathrm{N}-\mathrm{sp}^{2} \mathrm{C}\right)$ was found to be more abundant than the pyridine type $\left(\mathrm{N}-\mathrm{sp}^{3} \mathrm{C}\right.$ bonds) bonds at higher growth a temperature was showed in Fig. $2 b, d$, f.

\section{Raman spectroscopy}

Raman spectroscopy is a frequently used technique to characterize the carbon nanomaterials. It is a non-destructive tool applied to the identification and characterization of a wide variety of carbon nanomaterials, and the technique has been shown to be an excellent tool to investigate the crystallinity and graphitic nature of CNTs. Raman spectra of CNTs showed a strong band around $1,585 \mathrm{~cm}^{-1}$, originating from the Raman active E2 $\mathrm{g}$ mode which is referred to as the $\mathrm{G}$ band and a $\mathrm{D}$ band at about $1,350 \mathrm{~cm}^{-1}$ which is normally explained as a disorder-induced feature. The $\mathrm{D}$ band originates from defects in the curved graphene sheets. In N-CNTs, the formation of pentagons and heptagons due to the doping of $\mathrm{N}$ atoms leads to a distortion in the graphite sheets. Thus, the intensity ratio of the $D$ to $G$ band in the N-CNTs will be affected by the number of defects originating from nitrogen atom incorporation in hexagonal network. As the concentration of the $\mathrm{N}$ atoms increases, the $\mathrm{D}$ band becomes stronger and broader.

The intensity ratio $I_{\mathrm{d}} / I_{\mathrm{g}}$ is thus useful in estimating the defect concentration of $\mathrm{N}$ in the N-CNTs; as the $I_{\mathrm{d}} / I_{\mathrm{g}}$ ratio decreases the CNTs will have a more ordered structure. The relative intensity ratio of the $\mathrm{D}$ band to $\mathrm{G}$ band (i.e., $I_{\mathrm{d}} /$ $I_{\mathrm{g}}$ ) can be used as a rough measure of N-CNTs quality. Nitrogen content will have the profound effect on the $\mathrm{G}$ band (Liu et al. 2010; Yang et al. 2005). Raman analysis of $\mathrm{N}-\mathrm{CNTs}$ grown at different growth temperature was illustrated in Fig. 3a, b showed the ratio of D and $\mathrm{G}$ band and the in-plane crystallite size of the samples. N-CNTs, differences in chemical bond lengths and atomic masses as well as the formation of pentagons due to the doping of $\mathrm{N}$ atoms lead to local distortion in the graphite sheets. The $I_{\mathrm{d}} / I_{\mathrm{g}}$ ratio gives the measure of crystallinity of the CNTs. The lower its value, higher is the crystallinity. The in-plane crystallite size strongly depends on $I_{\mathrm{d}} / I_{\mathrm{g}}$ ratio (Cancardo et al. 2006) and it will decrease with the increase in $I_{\mathrm{d}} / I_{\mathrm{g}}$ ratio. In-plane crystallite size can be calculated as

$L_{\mathrm{a}}=\left(2.4 \times 10^{-10}\right) \lambda^{4} /\left(I_{\mathrm{d}} / I_{\mathrm{g}}\right)$

where $L_{\mathrm{a}}=$ in-plane crystallite size $(\mathrm{nm}), \lambda=$ wavelength of the laser source (nm).
Field emission study

Field emission consists in the extraction of electrons from a conducting solid under the application of an electric field and the mechanism for the emission of electron was illustrated in Fig. 4a. Unlike thermion emission, no heat is required for obtaining field emission. Very high electric fields are, however, required for the electrons to tunnel through the surface potential barrier. When the solid is shaped as the tip, the electric fields lines are concentrated around the tip and the local electric field is enhanced. This geometrical enhancement of the electric field is used in field emitters to allow extraction of electrons from sharp tips at relatively low macroscopic electric fields. Maximizing the current output for a given applied voltage requires a material with a low work function that is shaped as sharp as possible to offer the highest field enhancement factor. The emission characteristics of emitters are strongly dependent on the tip radii, and preservation of the emission characteristics requires the tip sharpness remain unaltered. Figure $4 \mathrm{~b}$ showed the measured $I-V$ characteristics of the carbon nanotube pillar arrays. It indicated that the emission current increases monotonically with the applied field and the turn-on field value required to obtain $2 \mu \mathrm{A}$ current for cold cathode was observed at $\sim 500 \mathrm{~V}$. Good field emission from the doped CNTs is attributed to the presence of nitrogen atoms in the hexagonal graphite network. In doping mechanism, the nitrogen substitutes the carbon atom on the graphite layer and modifies the conductance property of N-CNTs by donating electrons. The iron particles were used as the catalyst for the growth of the CNTs and were encapsulated. These catalyst particles may also contribute additional electron density that leads to improved field emission current. Field emission study of as-grown nitrogen-doped carbon nanotubes suggests that they are good emitters with a turn-on and threshold field of 0.9 and $1.6 \mathrm{~V} / \mu \mathrm{m}$, respectively. The maximum current density was observed to be $18.8 \mathrm{~mA} / \mathrm{cm}^{2}$ at electric field of $2.1 \mathrm{~V} / \mu \mathrm{m}$. The Fowler-Nordheim model was used to describe the field emission effectiveness. Figure $4 \mathrm{~b}$ shows the corresponding Fowler-Nordheim (F-N) plots. The F-N plots of the N-CNTs arrays almost straight line, showing that the electron emission is due to the tunneling effect. According to the $\mathrm{F}-\mathrm{N}$ theory, the slope of the $\mathrm{F}-\mathrm{N}$ plot is $-6.83 \times 10^{9} \varnothing^{3 / 2} \mathrm{~d} / \beta$, where $\varnothing$ is the work function of the emitter and $d$ is the electrode distance and $\beta$ is the geometrical enhancement factor. The field amplification factor is defined as $F=\beta V / d$, where $F$ is the field just above the surface of the tip, and $V$ the applied voltage. It is considered that the good field emission performance of doped nanotube is due to the presence of lone pairs of electrons on nitrogen atom that supplies more electrons to the conduction band and also the increased local density of states in 

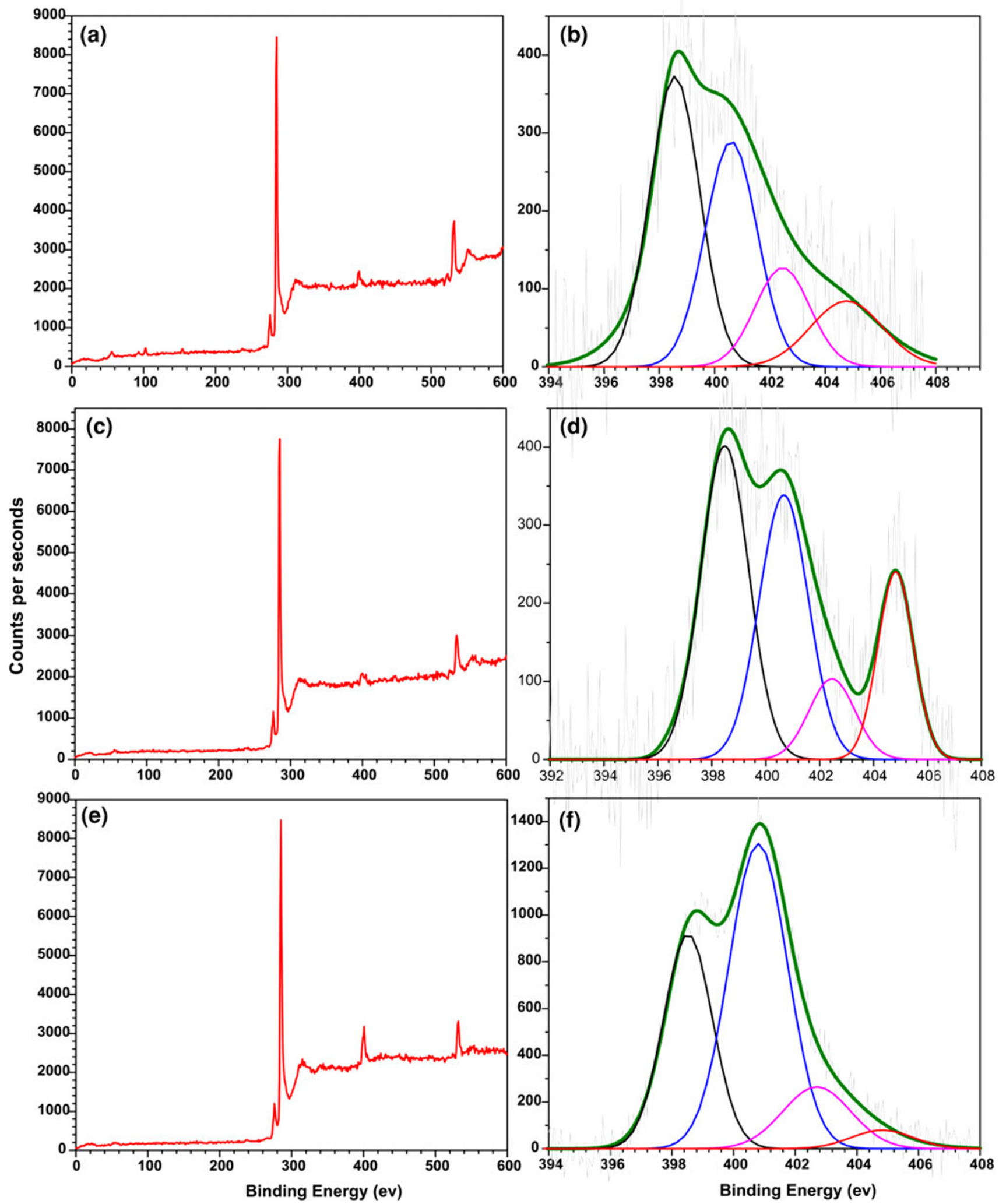

Fig. 2 XPS wide spectrum and N1s narrow spectrum for NCNT arrays grown at different growth temperature at $800{ }^{\circ} \mathrm{C}(\mathbf{a}, \mathbf{b})$, at $850{ }^{\circ} \mathrm{C}$ $(\mathbf{c}, \mathbf{d})$ and at $900{ }^{\circ} \mathrm{C}(\mathbf{e}, \mathbf{f})$ 

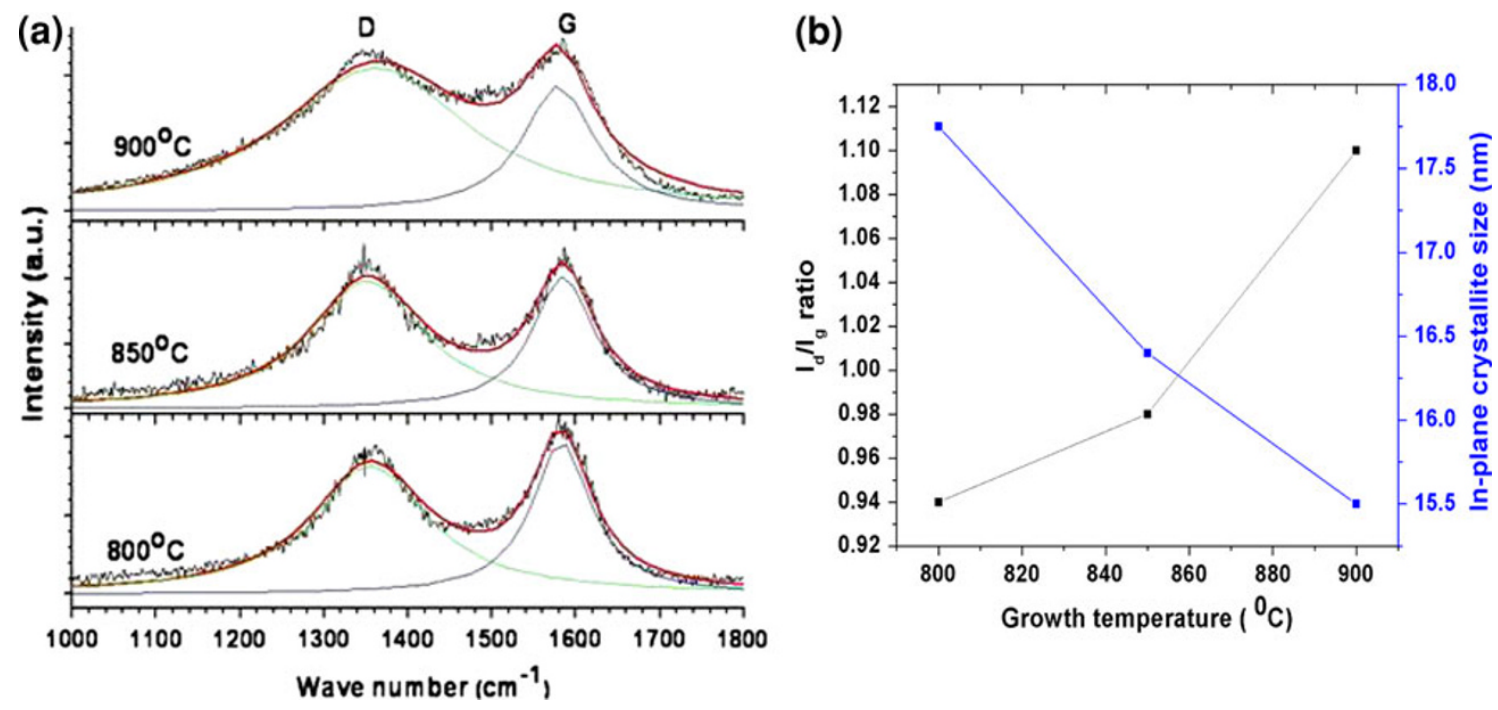

Fig. 3 Typical Raman spectrum (a) and the in-plane crystallite size (b) of N-CNT arrays grown at different growth temperatures

Fig. 4 Field emission properties of N-CNT arrays (a) field emission mechanism (b) $I-V$ characteristics and $\mathrm{F}-\mathrm{N}$ plot (inset) for the N-CNTs arrays grown at $900{ }^{\circ} \mathrm{C}$ (a)

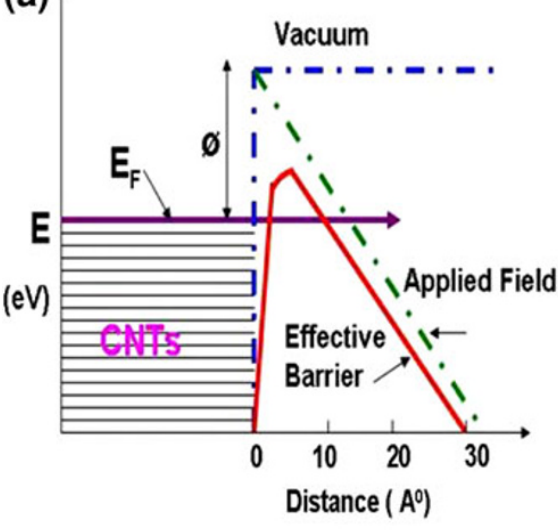

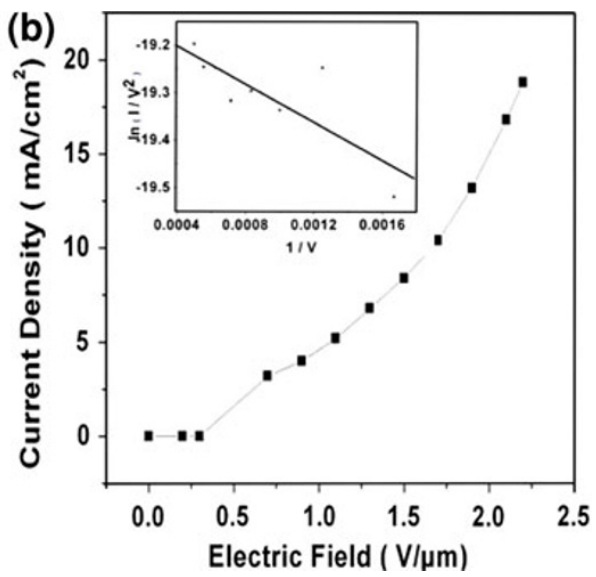

the conduction band close to the Fermi level through nitrogen incorporation.

The nitrogen incorporation into the nanotubes hexagonal network enhances the electrical conductance of nanotube films which in turn lowers the turn-on field and threshold fields in the field emission study. When the graphite sheet of CNTs bends (internal capping), which resulted in the deterioration in crystallinity of CNTs, the atomic bonding must lose some of its $\mathrm{sp}^{2}$ character and gain some $\mathrm{sp}^{3}$ character. The transformation of $\mathrm{sp}^{2}$ into $\mathrm{sp}^{3}$ characteristics will enhance the emission properties by lowering of the effective work function, increase of the field enhancement factor and the number of the emission sites. Along with the lone pairs of electrons supplied by the nitrogen atom, the curve at the tip of the N-CNTs consist of defects responsible for the concentration of degree of states (DOS) at the tip and are the prime source to improve the field emission. The disordered structure of the tip and the high defect density may also have an influence on the field emission properties.

\section{Conclusions}

Nitrogen-content-modulated growth of CNTs was developed by controlling the growth temperature to tailor the field emission properties. The additional lone pair of electrons on nitrogen atom that acts as a donor with respect to the delocalized $\pi$ system of the hexagonal framework which can enhance the conducting property of doped CNTs in turn will enhance the emission properties by lowering the effective work function. The maximum current density was observed to be $18.8 \mathrm{~mA} / \mathrm{cm}^{2}$ at $2.1 \mathrm{~V} / \mu \mathrm{m}$ electric field and linear Fowler-Nordheim plot verifies that such field electron emission characteristics can be mainly caused by the quantum tunneling effect. 
Acknowledgments We are thankful to director Dr. G. Sundararajan for permitting to publish these results. We are also thankful to G.V. Reddy and K. Subbarao for their technical support in present work. We are thankful to Dr. B.V. Sharada for Raman spectroscopy, Dr. Neha Hebalkar for XPS analysis, Mr. L. Venkatesh for SEM micrographs.

Open Access This article is distributed under the terms of the Creative Commons Attribution License which permits any use, distribution, and reproduction in any medium, provided the original author(s) and the source are credited.

\section{References}

Cancardo LG, Takai K, Enoki T, Endo M, Kim YA, Mizusaki H, Jorio A, Coelho LN, Magalhaes-Paniago R, Pimenta MA (2006) General equation for the determination of the crystallite size La of nanographite by Raman spectroscopy. Appl Phys Lett 88: $163106(1-3)$

Choi WB, Chung DS, Kang JH, Kim HY, Jin YW, Han IT, Lee YH, Jung JE, Lee NS, Park GS, Kim JM (1999) Fully sealed, highbrightness carbon-nanotube field-emission display. Appl Phys Lett 75(20):3129-3131

Czerw R, Terrones M, Charlier JC, Blasé X, Foley B, Kamalakaran R, Grobert N, Terrones H, Tekleab D, Ajayan PM, Blau W, Ruhle M, Carroll DL (2001) Identification of electron donor states in $\mathrm{N}$-doped carbon nanotubes. Nano Lett 1(9):457-460

Ganesan Y, Peng C, Lu Y, Ci L, Srivastava A, Ajayan PM, Lou J (2010) Effect of nitrogen doping on the mechanical properties of carbon nanotubes. ACS Nano 4(12):7637-7643

Ghosh K, Kumar M, Maruyama T, Ando Y (2010) Tailoring the field emission property of nitrogen doped carbon nanotubes by controlling the graphitic/pyridinic substitution. Carbon 48(1):191-200
Hafner JH, Cheung CL, Leiber CM (1999) Growth of nanotubes for probe microscopy tips. Nature 398:761-762

He M, Zhou S, Zhang J, Liu Z, Robinson C (2005) CVD growth of $\mathrm{N}$-doped carbon nanotubes on silicon substrates and its mechanism. J Phys Chem B 109(19):9275-9279

Ibrahim EMM, Khavrus VO, Leonhardt A, Hampel S, Oswald S, Rummeli MH, Buchner B (2010) Synthesis, characterization, and electrical properties of nitrogen doped single walled carbon nanotubes with different nitrogen content. Diam Relat Mater 19:1199-1206

Jang JW, Lee CE, Lyu SC, Lee TJ, Lee CJ (2004) Structural study of nitrogen doping effects in bamboo shaped multiwalled carbon nanotubes. Appl Phys Lett 84(15):2877-2879

Lim SH, Elim HI, Gao XY, Wee ATS, Ji W, Lee JY, Lin J (2006) Electronic and optical properties of nitrogen doped multiwalled carbon nanotubes. Phys Rev B 73(4):45402(1-6)

Liu H, Zhang Y, Li R, Sun X, Silets SD, Rachid HA, Jaidann M, Lussier LS (2010) Structural and morphological control of aligned nitrogen doped carbon nanotubes. Carbon 48:1498-1507

Niu C, Sickel EK, Hoch R, Moy D, Tennent H (1997) High power electrochemical capacitors based on carbon nanotube electrodes. Appl Phys Lett 70(11):1480-1483

Reddy ALM, Shaijumon MM, Gowda SR, Ajayan PM (2009) Coaxial $\mathrm{MnO}_{2} /$ carbon nanotube array electrodes for high-performance lithium batteries. Nano Lett 9(3):1002-1006

Terrones M, Ajayan PM, Banhart F, Blasé X, Carroll DL, Charlier JC, Czerw R, Foley B, Grobert N, Kamalakaran R, Redlich PK, Ruhle M, Seeger T, Terrones H (2002) N-doping and coalescence of carbon nanotubes: synthesis and electronic properties. Appl Phys A 74(3):355-361

Yang QH, Hou PX, Unno M, Yamauchi S, Saito T, Kyotani T (2005) Dual Raman features of double coaxial carbon nanotubes with N-doped and B-doped multiwalls. Nano Lett 5(12):2465-2469 